\title{
Multifocal skeletal tuberculosis: A case report
}

\author{
LIANG ZHANG, JINGCHENG WANG, XINMIN FENG, YUPING TAO, \\ JIANDONG YANG, SHENFEI ZHANG and JUN CAI
}

\begin{abstract}
Department of Orthopedics, Clinical Medical College of Yangzhou University, Subei People's Hospital of Jiangsu, Yangzhou, Jiangsu 225001, P.R. China
\end{abstract}

Received December 30, 2014; Accepted January 11, 2016

DOI: $10.3892 /$ etm.2016.3032

\begin{abstract}
Tuberculosis (TB) of the musculoskeletal system is a rare clinical condition. Multifocal bone involvement is extremely rare and difficult to recognize. Thus, due to the diverse and atypical clinical manifestations of multifocal skeletal TB, the disease is easy to misdiagnose. In the present study, a rare case of atypical disseminated multifocal skeletal TB was reported, which exhibited uncommon findings in radiological images that were more suggestive of a hematological malignancy or metastatic disease. In conclusion, the diagnosis of this condition by conventional diagnostic methods is challenging. The importance of CT-guided needle biopsy and open biopsy in the diagnosis of skeletal TB was emphasized.
\end{abstract}

\section{Introduction}

Tuberculosis (TB) remains a major cause of morbidity and mortality in developing countries affecting millions of people worldwide $(1,2)$. Skeletal TB, constituting $10-20 \%$ of all the extrapulmonary TB (ETB), is a well-recognized clinical condition that is easily diagnosed and managed with an excellent outcome (2). However, the occurrence of multifocal skeletal involvement, which is defined as osteoarticular lesions that occur simultaneously at two or more locations in the skeletal system, is exceptional and constitutes $<5 \%$ of all skeletal TB cases, even in countries where TB is endemic (3-6).

Radiological examinations, including conventional roentgenography, magnetic resonance imaging (MRI) and computed tomography (CT) scans, can be used to assist the diagnosis of multifocal skeletal TB $(4,5,7)$. However, the diagnosis and treatment of multifocal skeletal TB are frequently delayed due to the rarity and vague symptoms of the disease, thus allowing progression to severe deformities and functional deficits $(3,5,6)$.

Correspondence to: Professor Xinmin Feng, Department of Orthopedics, Clinical Medical College of Yangzhou University, Subei People's Hospital of Jiangsu, 98 Nantong West Road, Yangzhou, Jiangsu 225001, P.R. China

E-mail: 17374769@qq.com

Key words: skeletal tuberculosis, multifocal, vertebral
In the present study, a case of atypical disseminated multifocal skeletal TB is presented. Unusual radiological images were observed, which were more compatible with a hematological malignancy or metastatic disease rather than an infectious disease. Despite widespread osteoarticular involvement, the outcome of the patient was favorable following anti-TB treatment.

\section{Case report}

A 19-year-old male patient was admitted to Subei People's Hospital of Jiangsu (Yangzhou, China) on 5th September 2011 with a 2 month history of recurrent fever and intermittent thoracic back pain. The recurrent fever did not occur at specific times and the temperature did not exceed $38.0^{\circ} \mathrm{C}$. The patient also reported limited mobility, decreased appetite and weight loss, but did not experience chills or night sweats. There was no history of exposure to TB or of a chronic cough. The patient presented a normal general physical condition, with a body temperature of $37.3^{\circ} \mathrm{C}$. A physical examination revealed slight tenderness over the mid-thoracic spine, lumbar spine and left sacroiliac joint. No paraspinal swelling or spasms were observed, while the sensation, muscle strength and muscle tone of the lower extremities were normal. Laboratory examinations showed a normal white blood cell count $\left[6.6 \times 10^{9} / 1\right.$; normal range (NR), 3.5-9.5x $10^{9} / 1$ ] and normal levels of hemoglobin (120 g/l; NR, 110-160 g/l), human leukocyte antigen-B27 (78; $\mathrm{NR}, 0-145$ ) and rheumatoid factor (5.0 IU/ml; NR, 0-14 IU/ml). In addition, tests for autoantibodies, immunoglobulin, TB antibodies and tumor-associated antigens were negative. The erythrocyte sedimentation rate (ESR) was $75 \mathrm{~mm} / \mathrm{h}$, which was higher than the normal range $(0-15 \mathrm{~mm} / \mathrm{h})$, and the C-reactive protein level was normal (10 mg/l; NR, 0-10 mg/l). Human immunodeficiency virus (HIV) and purified protein derivative (PPD) tests were negative.

Further imaging analysis showed normal abdominal ultrasound findings and X-rays of the chest, thorax, lumbosacral region and pelvis. CT reconstruction of the sacroiliac joint indicated multiple round hypodense lesions with a hyperdense center, as well as some cortical destruction and soft tissue swelling in the lumbar and sacral vertebrae, and the left ilium regions (Fig. 1). A contrast-enhanced CT scan of the chest, with Omnipaque ${ }^{\mathrm{TM}}(1.5 \mathrm{ml} / \mathrm{kg}$; GE Healthcare Life Sciences, Shanghai, China) as the contrast enhancement agent, demonstrated an irregular patchy hypodense signal in the left lung 

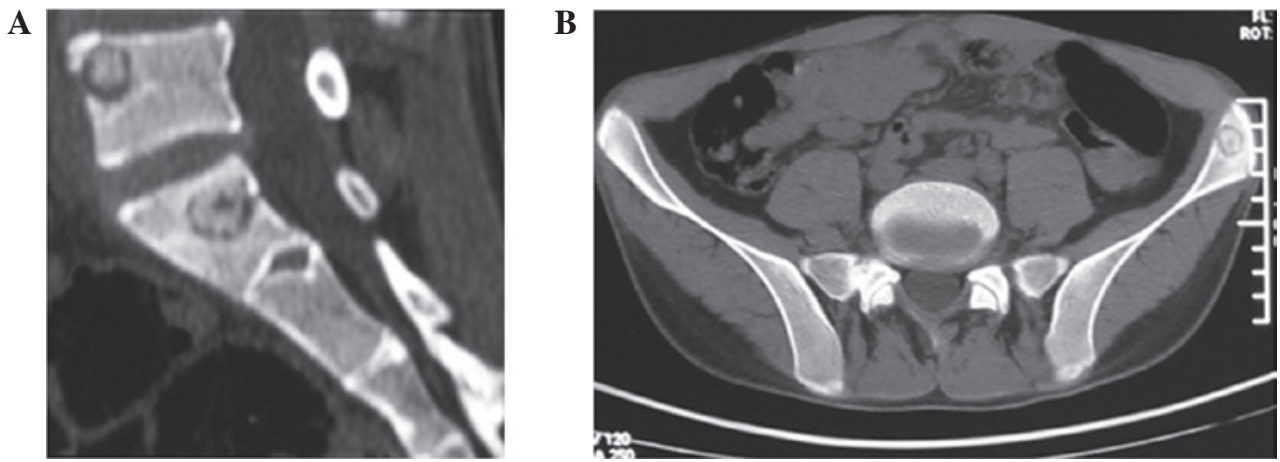

Figure 1. Computed tomography reconstruction of sacroiliac joint scan showing round hypodense lesions with central higher density and some cortical destruction in the (A) lumbar and sacral vertebrae and (B) left ilium.

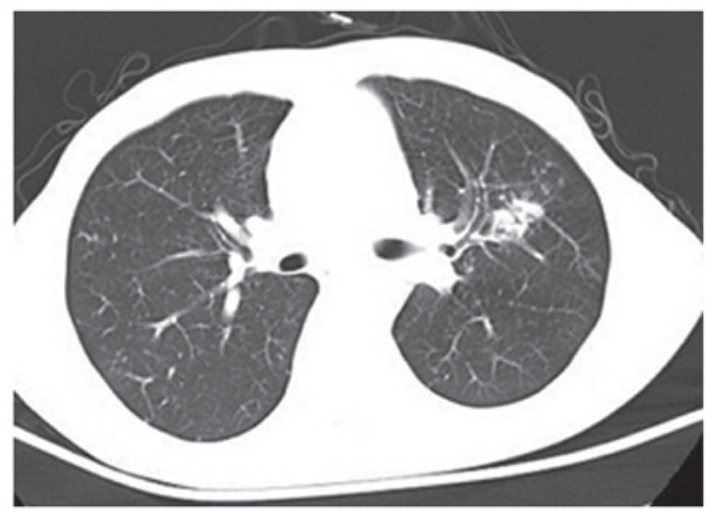

Figure 2. Contrast-enhanced chest computed tomography scans showing irregular patchy hypodense change in the left lung lobe with patchy translucent shadow inside.

lobe with a patchy translucent shadow inside, which was enhanced heterogeneously (Fig. 2). A chest CT scan showed multiple round hypodense lesions with higher central density in the thoracic vertebrae, ribs and sternum (Fig. 3). Furthermore, a CT reconstruction of the thoracic spine indicated bone destruction with partial cortical perforation (Fig. 4). MRI scans revealed multiple round ring signal shadows with a continuous finishing border from Th12 to S4 in the anterior, central and posterior elements, but not in the end plate. In addition, sagittal T1-weighted and T2-weighted images revealed a hypodense signal in the internal of the lesions and a slightly hypodense signal in the peripheral region, which was accompanied by soft tissue swelling, with visible ring enhancement and soft tissue enhancement following intravenous administration of gadolinium (GE Healthcare Life Sciences) (Fig. 5).

Based on the symptoms and all the aforementioned examinations, lymphoma, metastatic disease or bone TB were suspected. Since the CT and MRI findings did not allow for a final diagnosis, a CT-guided biopsy from the lesions in the Th7 vertebral, left iliac and adjacent left lung using an 8-gauge needle (Yangzhou City Jiangzhou Medical Instrument Co., Ltd., Yangzhou, China) was performed to confirm the diagnosis. However, no evidence of granulomata, necrosis, lymphoma or other malignancies was observed. Subsequently, an open biopsy was performed in order to establish a pathological diagnosis, during which fish-shaped soft tissue adhesion with surrounding tissues under the lamina of Th7 and local sequestrum formation were observed. Part of

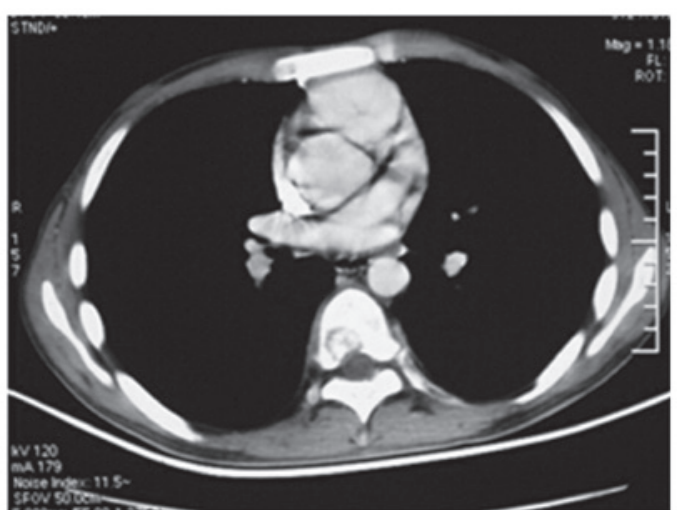

Figure 3. Computed tomography scan of the chest showing multiple round hypodense lesions with higher central density in the thoracic vertebrae, ribs and sternum.

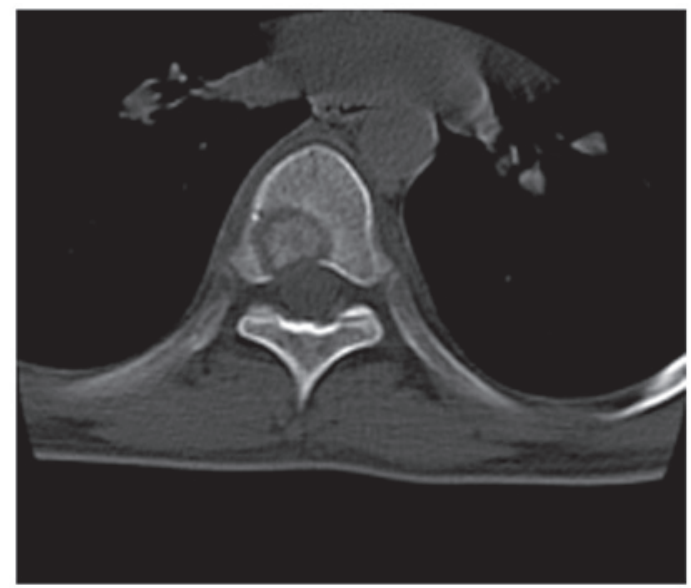

Figure 4. Computed tomography scan of the vertebral body of Th7 showing bone destruction with partial cortical perforation.

the surrounding soft tissues and bone lesions were removed for pathological examination and culturing. Upon hematoxylin and eosin staining of the tissue and visualization under the Olympus BX43 microscope (Olympus Corporation, Tokyo, Japan), a large amount of necrosis, epithelioid cells, Langerhans giant cells and dead bone were observed (Fig. 6), which are characteristics of TB $(2,5)$. The results of Acid-Fast Bacilli culturing (Sinobest Biotech Co., Ltd., Shanghai, China) were 

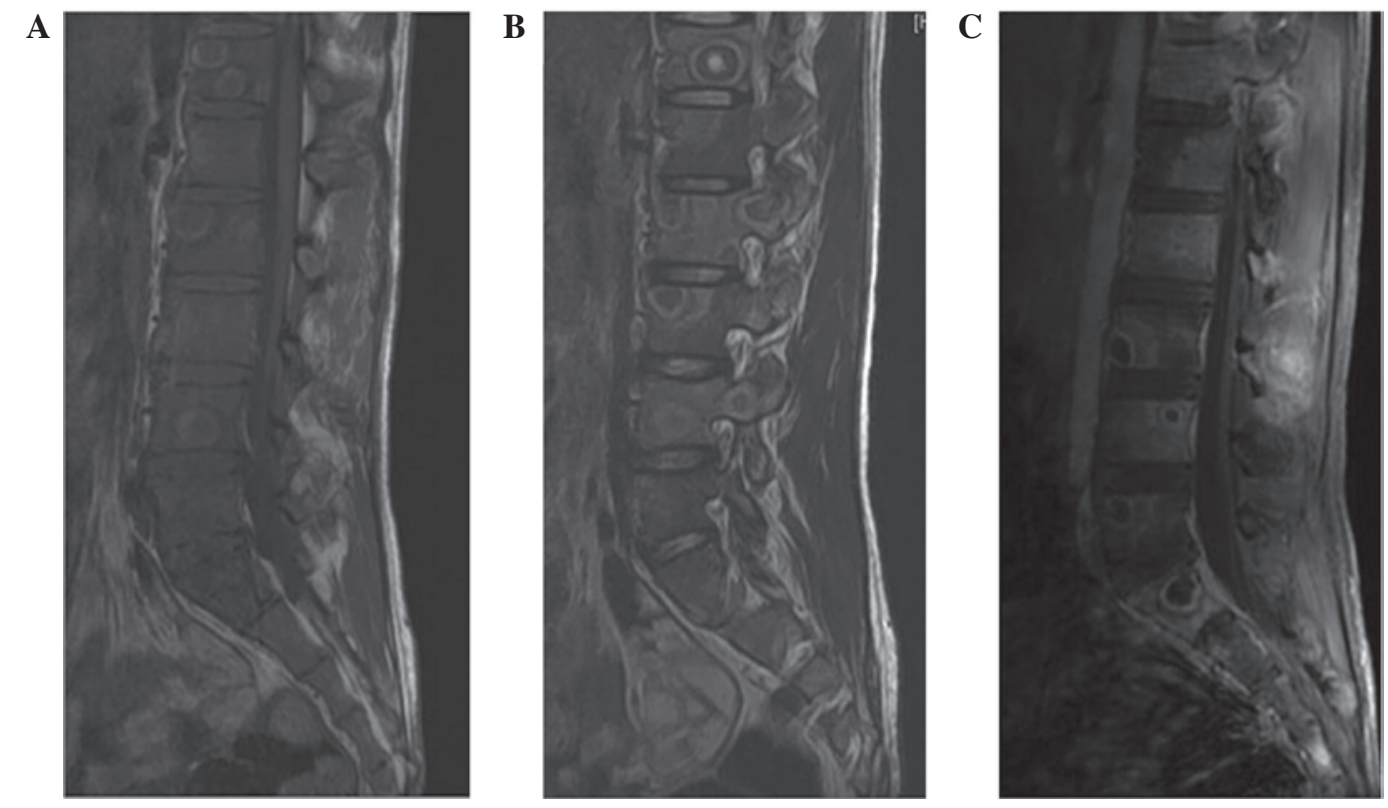

Figure 5. Magnetic resonance imaging scans showing multiple round ring signal shadows with a continuous finishing border from Th12 to S4. Sagittal (A) T1-weighted and (B) T2-weighted images revealed hypodense change in the internal region of the lesions and slightly hypodense change in the peripheral region, accompanied by some soft tissue swelling, (C) with evident ring enhancement and soft tissue enhancement, following administration of a contrast agent.

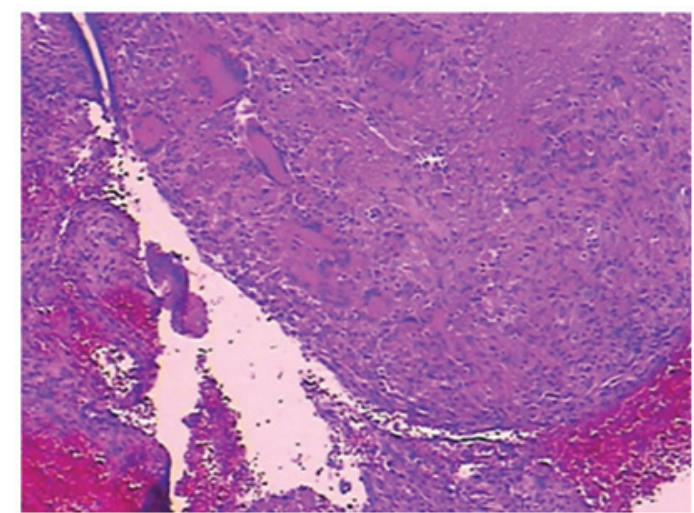

Figure 6. Histopathological examination showing a large amount of necrosis, epithelioid cells, Langerhans giant cells and dead bone (hematoxylin and eosin staining; magnification, $\mathrm{x} 200$ )

negative; however, a polymerase chain reaction performed by Shanghai Genechem Co., Ltd. (Shanghai, China) was positive for TB. Based on these observations, a diagnosis of multifocal skeletal TB was established.

The patient was discharged from the hospital on 27th September 2011. Postoperatively, the patient was administered a common quartet anti-TB chemotherapy regimen $(2,5)$, including isoniazid (300 mg/day), rifampicin (600 mg/day), pyrazinamide $(750 \mathrm{mg} /$ day $)$ and ethambutol $(750 \mathrm{mg} /$ day; all Shanghai Sine Pharmaceutical Co., Ltd., Shanghai, China) for 12 months. The patient's symptoms greatly improved after 1 month of anti-TB treatment and the ESR decreased to $65 \mathrm{~mm} / \mathrm{h}$. At the final follow-up on 11th March 2013, the patient was free of symptoms and the ESR had decreased to the normal value. MRI scans obtained following 20 months of anti-TB therapy showed that the range of the extensive abnormal signal intensity had become smaller, as compared with that before anti-TB treatment was initiated (Fig. 7).
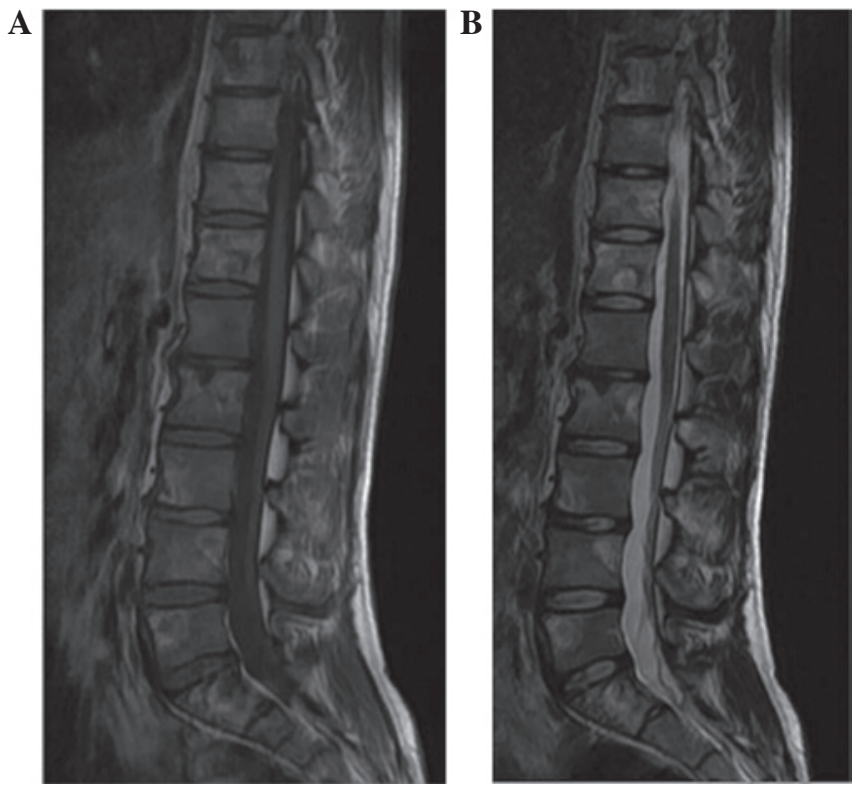

Figure 7. Magnetic resonance imaging scans at 20 months after antituberculosis therapy showed only a slightly smaller range of the extensive abnormal signal intensity on (A) T1-weighted (low-to-high unequal signal intensity) and on (B) T2-weighted images (high signal intensity), as compared with the findings before treatment.

Informed consent was obtained from the patient's family prior to the publication of the present case.

\section{Discussion}

TB remains a major cause of morbidity and mortality worldwide, and is responsible for $\sim 1.6$ million deaths per year $(1,4)$. According to the World Health Organization, $\sim 8.8$ million new cases of TB occur every year worldwide $(5,8)$. Multifocal skeletal TB has rarely been reported 
in non-immunocompromised patients and in patients with normal pulmonary findings, particularly in China $(5,9)$. The spine is the most commonly affected site, of which the upper lumbar and lower thoracic regions are the most frequently involved, comprising $50 \%$ of all multifocal skeletal TB cases (3-6). The average number of vertebrae that are affected by multifocal TB, as seen radiologically, is 3.0 in children and 2.5-3.8 in adults (10). In the present case, there were multiple round hypodense lesions in the ribs, sternum, multi-segment thoracic, multi-segment lumbar and sacral vertebrae, and ilium regions, accompanied by some adjacent soft tissue swelling.

A positive PPD test is a fundamental finding in TB patients; however, a negative test is not sufficient to exclude the disease. Although radiological findings do not firmly establish the diagnosis in the case of a negative PPD result, imaging remains the most useful diagnostic tool in such cases $(5,6,10)$. The characteristic findings of spinal TB include destruction of two adjacent vertebral bodies, destruction of the intervening disc, and the occurrence of paravertebral and epidural abscesses $(7,11)$.

Skeletal TB does not present any specific features on radiological images in the early stages of the disease, thus mimicking metastatic tumors or certain primary osseous lesions, such as eosinophilic granuloma, particularly if multiple destructive lesions are present $(5,7,12)$. Plain radiography is of little help unless the collapse of the disc margin or vertebra body appears (4-7). In addition, CT scans are unable to show the early alterations in the vertebral marrow and to differentiate an infectious disease from neoplastic involvement (4-7). By contrast, MRI has been shown to be a more sensitive and specific imaging modality that may guide the diagnosis at early stages of skeletal TB. The typical MRI patterns are abnormal signal intensities appearing hypointense or isointense on a T1-weighted image of the affected vertebral bodies and discs and hyperintense on a T2-weighted image of the osseous, and soft-tissue changes with heterogeneous enhancement of the vertebral body $(4-7,11)$. Overall, the sensitivity and specificity of MRI for TB may be 100 and $88.2 \%$, respectively $(5,7)$. In the present case, the MRI features included multiple round hypodense lesions with finishing borders in the thoracic, lumbar and sacral vertebrae, which was not sufficient evidence for the diagnosis of TB; on the contrary, these observations were suggestive of hematological malignancies, such as lymphoma or multiple myeloma, or metastatic diseases. The common characteristics of end-plate disruption and high signal intensity of the intervertebral disc were not demonstrated. Therefore, the presentation of the TB case reported in the current study is considered unusual.

To the best of our knowledge, the present study is the first to report an extremely rare case of multifocal osteoarticular TB with multiple round hypodense lesions and a surprisingly negative PPD test result. The ETB and disseminated forms of the disease are usually more frequent in certain patients, such as those with a much older age, malnutrition and hemodialysis, as well as immunocompromised and in particular HIV-infected individuals $(5,6)$. However, the current patient was a healthy 19-year-old male with no signs of systemic involvement and a negative HIV status. This demonstrated the diagnostic difficulties of the atypical forms of TB.
In patients with skeletal $\mathrm{TB}$, the onset of symptoms is generally insidious, without general alarming signs, such as fever, night sweats, toxicity or extreme weakness. This condition may mimic malignant diseases, both clinically and radiographically. Although $\mathrm{CT}$ and MRI are reliable techniques that help establish a diagnosis, biopsy or CT-guided needle aspiration remain essential for accurate diagnosis and adequate treatment. The advantage of needle aspiration is that it is a less invasive technique; however, only a small number of specimens can be obtained, particularly when the lesions are sclerotic, and thus the diagnosis rate is only $81.0 \%$ in children (13) and $91.3 \%$ in adults (14). Hao et al (15) concluded that the diagnostic accuracy of CT-guided thoracic spinal biopsy using a 16-gauge needle was $90.5 \%$ overall, as the diagnostic accuracy was significantly lower for the middle thoracic spine (90.0\%) compared with that for the lower spine (97.6\%), as well as lower for sclerotic lesions $(81.3 \%)$ compared with that for lytic lesions (96.4\%). Similarly, in the present study, the CT-guided aspiration using an 8-gauge needle was not able to confirm the diagnosis, possibly due to the limited amount of tissue acquired and the histological nature of the lesion itself, resulting in the use of open biopsy to establish the diagnosis.

Multidrug anti-TB therapy including isoniazid, rifampin, ethambutol and pyrazinamide advocated for an approximate duration of 12-18 months usually leads to complete resolution of TB $(2,5)$. Medical treatment is highly effective when administered appropriately, as performed in the present study.

The mean time to diagnosis is reported to be 16-19 months after the presentation of symptoms (11). Ringshausen et al (12) described the fatal case of a patient with spinal TB, who was mistakenly irradiated for suspected metastatic lung cancer of the spine. The authors claimed that the most common cause in delayed diagnosis is failure to consider this disease (12). Thus, TB should be considered in all patients with multiple round hypodense lytic lesions of the skeletal system, especially in countries endemic for TB, in order to avoid delays that may result in irreversible damage and in a high mortality rate.

In conclusion, the diagnosis of multifocal skeletal TB is difficult for various reasons, requiring careful consideration and numerous examinations. A high index of suspicion for the possibility of TB appearance has to be maintained in any patient with multiple round hypodense lytic lesions of the skeletal system in countries endemic for TB. In addition, MRI is particularly valuable in the diagnostic and follow-up procedures. However, confirmation of the diagnosis may only be possible following biopsy.

\section{References}

1. Dye C, Scheele S, Dolin P, Pathania V and Raviglione MC: Consensus statement. Global burden of tuberculosis: Estimated incidence, prevalence and mortality by country. WHO global surveillance and monitoring project. JAMA 282: 677-686, 1999.

2. Moon MS, Moon YW, Moon JL, Kim SS and Sun DH: Conservative treatment of tuberculosis of the lumbar and lumbosacral spine. Clin Orthop Relat Res 40-49, 2002.

3. Yilmaz MH, Kantarci F, Mihmanli I and Kanberoglu K: Multifocal skeletal tuberculosis. South Med J 97: 785-787, 2004.

4. Agarwal A, Khan SA and Qureshi NA: Multifocal osteoarticular tuberculosis in children. J Orthop Surg (Hong Kong) 19: 336-340, 2011. 
5. Hong L, Wu JG, Ding JG, Wang XY, Zheng MH, Fu RQ, Li WB, Peng WX, He WF and Sun QF: Multifocal skeletal tuberculosis: Experience in diagnosis and treatment. Med Mal Infect 40: 6-11, 2010

6. Marudanayagam A and Gnanadoss JJ: Multifocal skeletal tuberculosis: A report of three cases. Iowa Orthop J 26: 151-153, 2006

7. Danchaivijitr N, Temram S, Thepmongkhol K and Chiewvit P Diagnostic accuracy of MR imaging in tuberculous spondylitis. J Med Assoc Thai 90: 1581-1589, 2007.

8. Wright GD and Sutherland AD: New strategies for combating multidrug-resistant bacteria. Trends Mol Med 13: 260-267, 2007.

9. Zhang Y,Zhang Y and Ma J: The prospect of incidental detection of unsuspected skeletal tuberculosis by bone scintigraphy should not be overlooked. Clin Nucl Med 32: 435-439, 2007.

10. St Clair Strange FG: Current concepts review. Tuberculosis of bones and joints (78-A:288-298, Feb. 1996) by Watts and Lifeso. J Bone Joint Surg Am 80: 604, 1998.
11. Moore SL and Rafii M: Imaging of musculoskeletal and spinal tuberculosis. Radiol Clin North Am 39: 329-342, 2001.

12. Ringshausen FC, Tannapfel A, Nicolas V, Weber A, Duchna HW, Schultze-Werninghaus $\mathrm{G}$ and Rohde G: A fatal case of spinal tuberculosis mistaken for metastatic lung cancer: Recalling ancient Pott's disease. Ann Clin Microbiol Antimicrob 8: 32 , 2009.

13. Ballah D, Nijs E, Keller MS, Zhu X, Krishnamurthy G and Cahill AM: Percutaneous CT-guided vertebral bone biopsy in children. Pediatr Radiol 43: 582-588, 2013.

14. Poyanli O, Akan K, Unay K and Tangay C: CT-guided percutaneous transpedicular biopsy for the diagnosis of vertebral lesions. Acta Orthop Belg 74: 503-506, 2008.

15. Hao DJ, Sun HH, He BR, Liu TJ, Jiang YH and Zhao QP Accuracy of CT-guided biopsies in 158 patients with thoracic spinal lesions. Acta Radiol 52: 1015-1019, 2011. 